\title{
Manipulation of the Calibre of Air Weapon- A Case Study
}

\author{
Murthy SS ${ }^{*}$ and Rajkumar G² \\ ${ }^{1}$ Assistant Director (Ballistics), Central Forensic Science Laboratory, Ramanthapur, \\ Hyderabad, India \\ ${ }^{2}$ Senior Scientific Assistant (Ballistics), Central Forensic Science Laboratory, \\ Ramanthapur, Hyderabad, India
}

Case Report

Volume 3 Issue 2

Received Date: September 10, 2018

Published Date: October 26, 2018

DOI: $10.23880 /$ ijfsc-16000141

*Corresponding author: Dr. Murthy SS, Assistant Director (Ballistics), Central Forensic Science Laboratory, Ramanthapur, Hyderabad, India, Tel: 919989839067; Email: sistlasrinivasmuthy@hotmail.com

\section{Abstract}

Bombay customs department seized air rifles of different calibre. In one of the air rifles (Evanix, Rainstorm) the calibre was mentioned to be .177 " / $4.5 \mathrm{~mm}$ and the same was sent to the laboratory for Forensic examination to ascertain its original calibre. The image processing and the pattern recognition technique, i.e image sharpening \& edge detection, noise removal, pattern recognition and then, finally image recognition revealed the original calibre of the air rifle. The Gaussian filter was applied for the enhancement of the numbers [1]. Circles and straight lines are a common geometric structure of interest in computer vision applications. Hough transformation is used to locate circles and straight lines. This study also deals with The Arms Act 1959 read with the Arms Rules, New Delhi, 15th July, 2016, so as to understand the basic reasons for its tampering and manipulation.

Keywords: Air; Weapon

\section{Introduction}

The (Evanix, Rainstorm) air rifle, as shown in the Figure 1, was received in the laboratory. During the forensic examination, it was observed that the calibre was mentioned to be $.177 " / 4.5 \mathrm{~mm}$ on the right side of the rifle, as shown in Figure 2. The pellets of .177"/ $4.5 \mathrm{~mm}$ calibre from the laboratory stock were tried for chambering in the suspected air rifle and found that these pellets are under sized with respect to the chamber dimensions and hence the pellets passed through the barrel, which created doubt about its original calibre. The suspicion raised further and .22 " / $5.5 \mathrm{~mm}$ calibre pellet, was tried for chambering, as shown in Figure 3. The difference between the pellets of .177"/ $4.5 \mathrm{~mm}$ calibre and $.22 "$ / $5.5 \mathrm{~mm}$ calibre is shown in Figure 4. The .22"/
$5.5 \mathrm{~mm}$ calibre pellets could be successfully chambered and test fired. Hence, it was confirmed that the calibre of the rifle was suppressed and manipulated.

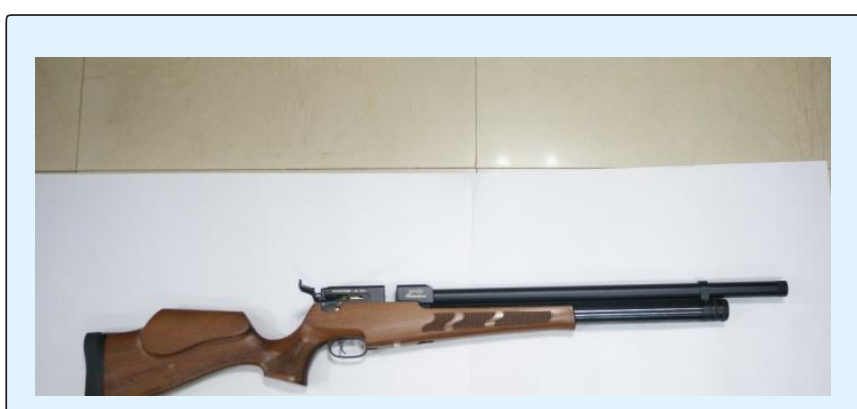

Figure 1: Evanix, Rainstorm air rifle. 


\section{International Journal of Forensic Sciences}

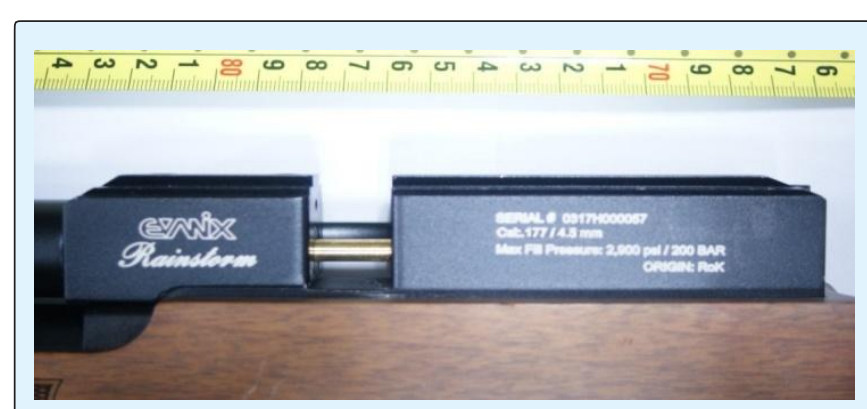

Figure 2: The serial number, the visible calibre and the max fill pressure of the Evanix, Rainstorm air rifle.

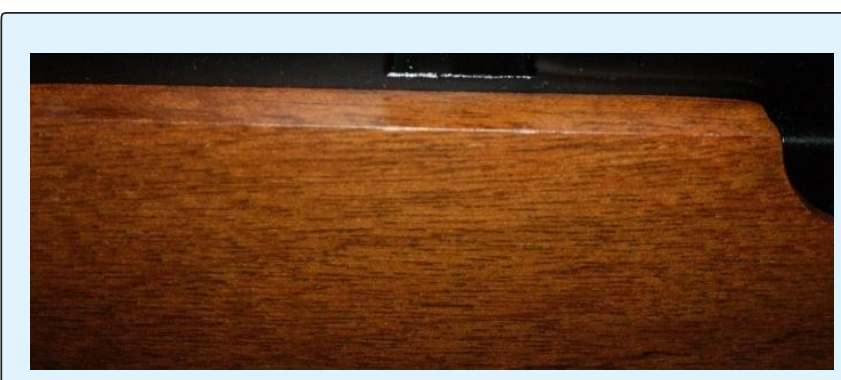

Figure 3: .22" / $5.5 \mathrm{~mm}$ calibre pellet to be chambered in the Evanix, Rainstorm air rifle.

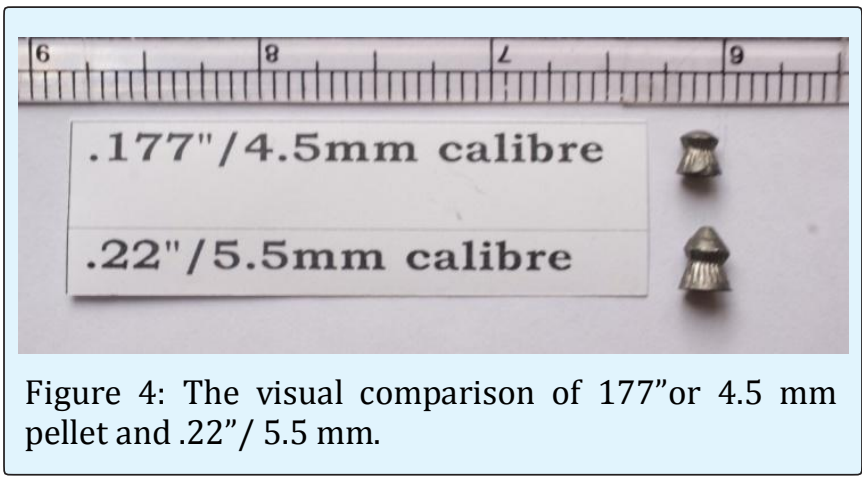

\section{Materials and Methods}

The image processing and the pattern recognition technique was applied to find out the genuine calibre of the rifle which was suppressed and manipulated. The process of filtering was applied over the image. Filtering is as an operation of removing frequency components from an image. The selection criteria depend on the filter.

a. A Low pass filter removes high frequency components (edges), and

b. A High pass filter removes low frequency components (slow changes in the image)
The effect of filtering can be seen by its effect on the Fourier spectrum where the pixels that correspond to the filtered frequencies are 'blackened' out. This effect can be seen in the illustration that follows. A Gaussian filter is a linear filter. It's usually used to blur the image or to reduce noise. If we use two of them and subtract, we can use them for "unsharp masking" (edge detection). The Gaussian filter alone will blur edges and reduce contrast. The Median filter is a non-linear filter that is most commonly used as a simple way to reduce noise in an image. Its claim to fame (over Gaussian for noise reduction) is that it removes noise while keeping edges relatively sharp. Gaussian filters are ideal to start experimenting with filtering because their design can be controlled by manipulating just one variable- the variance. Gaussian filter function with reference to the (x, y) co-ordinates is defined as [1]

$$
G(x, y)=\frac{1}{2 \pi \sigma^{2}} e^{-\frac{x^{2}+y^{2}}{2 \sigma^{2}}}
$$

Eqn. 1.1

The value of the sigma (the variance) as shown in equation 1.1, corresponds inversely to the amount of filtering, smaller values of sigma means more frequencies are suppressed and vice versa. The filtering process was applied on the figure 2. After filtering and noise removal, the image could be retrieved by recognizing its pattern. The pattern now, revealed about the different data as shown in figure 5 .

\section{SERIAL \# $0317 H 000057$ Calt.1Th/KG5imm Max Fill Preseure: 2.900 del / 200 BAR}

Figure 5: The image processing and the pattern recognition technique revealed that beneath the caliber .177 "or $4.5 \mathrm{~mm}$ on the air rifle .22 " / $5.5 \mathrm{~mm}$ is inscribed.

\section{Results and Discussion}

It was observed that beneath the calibre .177 "or 4.5 $\mathrm{mm}$ on the air rifle $.22^{\prime \prime} / 5.5 \mathrm{~mm}$ was found to be inscribed as shown in the Figure 5. Hence, the genuine calibre is .22" / $5.5 \mathrm{~mm}$ not the .177 " or $4.5 \mathrm{~mm}$. 


\section{International Journal of Forensic Sciences}

The Gazette Of India : Extraordinary [Part Ii-Sec. 3(I)], Ministry of Home Affairs Notification, The Arms Rules, New Delhi, 15th July, 2016 clearly states about the manufacture and sale of air weapons, which is as under.

i. The sale or transfer of air weapons including air rifles and air guns having muzzle energy more than 20 joules or $15 \mathrm{ft}$. lbs. and/or bore more than .177" or $4.5 \mathrm{~mm}$ and paintball markers or guns having muzzle energy not exceeding $90 \mathrm{~m} / \mathrm{s}$ or $300 \mathrm{ft} / \mathrm{s}$ (300 fsp), shall be made only through an authorised arms and ammunition dealer to a valid arms license holder.

ii. Sale or transfer by the authorised arms and ammunition dealer or the air weapon dealer of air weapons including air rifles and air guns having muzzle energy less than 20 joules or $15 \mathrm{ft}$. lbs. and/or bore less than .177" or $4.5 \mathrm{~mm}$ shall be made against obtaining of an identification and residence proof from the buyer thereof and against issuance of an invoice containing the said particulars at the time of sale [2].

\section{Conclusion}

The image processing techniques revealed that the calibre beneath.177" or $4.5 \mathrm{~mm}$ on the air rifle is .22"/ 5.5 $\mathrm{mm}$. Therefore, the original calibre is .22 " / $5.5 \mathrm{~mm}$ not the .177 "or $4.5 \mathrm{~mm}$. The following changes have been brought in the licensing regime for Air Guns in the Arms Rules, 2016. Air guns fall under the definition of firearms under clause (e) of subsection (1) of section 2 of the Arms Act, 1959 and were included under clause (d) of Category III in Schedule I of the Arms Rules, 1962. However, the air guns had been exempted from the licensing 11 requirements as per specifications contained in entry 3 of GSR 991 dated 1.10.1962. The Arms Rules, 2016 have brought in two categories of air weapons in clause (f) of category III of Schedule I of the rules, namely - (f) Air weapons including air rifles and air guns (i) having muzzle energy exceeding 20 joules or $15 \mathrm{ft}$. lbs. and/or bore exceeding 0.177 "or $4.5 \mathrm{~mm}$ (ii) having muzzle energy less than 20 joules or $15 \mathrm{ft}$. lbs. and /or bore less than 0.177 "or $4.5 \mathrm{~mm}$ Air weapons falling under category III(f)(i) shall be subject to the licensing requirements like any other firearm and shall be subject to the restriction of maximum three (3) firearms as specified in section 3 of the Arms Act, 1959 and can be kept and sold only through authorized arms and ammunition dealers licensed under the Arms Rules, 2016, holding a license in Form VIII. Air weapons falling under category III (f) (ii) shall not be subjected to the licensing requirements. Such air weapons may be kept and sold only through Air Weapon dealers holding a license in Form VIII-A under the Arms Rules, 2016 after obtaining identity and residence proof from the buyer. Manufacturing of all types of air weapons have been brought under the licensing regime and can be manufactured after obtaining a license in Form VII-C under the Arms Rules, $2016[3,4]$.

The air weapons could be dangerous in the close range firing phenomenon depending upon the (i) velocity of the projectile,(ii) weight of the projectile (iii) shape of the projectile, (iv) calibre of the projectile, (v) yawing, (vi) remaining kinetic energy of the projectile while entering the target, and (vii) vital parts of the target (either on the human and/or on animal) where projectile hits i.e the combination firearm, ammunition and nature of the target.

Therefore, the calibre of the air weapons and/or muzzle energy should be taken into consideration and examined very carefully so that no one can escape from the clutches of the law of the land, i.e. from the purview of The Arms Act 1959 read with Arms rule 2016.

\section{Acknowledgement}

The authors wish to thank to the Director, Central Forensic Science Laboratory, Ramanthapur, Hyderabad, India for conducting the test firings \& analysis in the laboratory and allowing this paper for publication.

\section{References}

1. https://www.cs.auckland.ac.nz/courses/compsci373 s1c/PatricesLectures/Gaussian\%20Filtering_1up.pdf

2. http://www.maharifle.org/Arms\%20Rules\%202016 $\% 20$ Notification $\% 20$ English.pdf.

3. https://mha.gov.in/sites/default/files/FAQArmsRule 2016andNDAL.pdf.

4. Murhty SS (2004) Some Studies on Image Processing and Pattern Recognition techniques for Identification of Small arms and Ammunition.

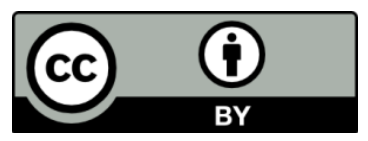

Murthy SS and Rajkumar D. Manipulation of the Calibre of Air Weapon- A Case Study. Int J Forens Sci 2018, 3(2): 000141. 